\section{CASE OF TRIPLE BIRTH FOLLOWED BY PUERPERAL ECLAMPSIA.}

By PERCY BOULTON, M.D., M.R.C.P. LOND., PHYSICIAN TO THE SAMARITAN FREE AND QUEEN CIIARLOTTL S HOSPITALS.

ON Sept. 21st I was asked to see A. O_-, who had been delivered the day previously of triplets, and was in strong convulsions. Excepting its plurality, the labsur had been normal. The large single placenta had come away naturally, and the uterus contracted well without any excessive hremorrhage. The patient was with difficulty kept in bed during the fits by two women in attendance, and in spite of a clothes-peg, which had been used as a gag, shehad bitten her tongue badly. She was pale, unconscious, foaming at the mouth, the pupils were dilated, the skin cool, and the legs much swollen. I was told that before the fits she had complained of severe headache, and that she had passed very little urine. In addition to the increased arterial tension during pregnancy, the enormous size of the uterus during triple pregnancy causes pressure on the large abdominal vessels, on the bladder, thereby diminishing its capacity, and to some extent on the ureters, preventing free escape of urine into the bladder-all tending to renal congestion and uræmic eclampsia. This patient, however, had no symptoms of convulsions till after confinement, when the pre-existing pressure had been removed, and therefore it was a question whether the fits were uræmic or not. The swollen extremities showed that although direct pressure bad been removed, the circulation had not recovered itself; and it is probable that the immediate result of delivery is to add to the troubles of renal incompetence, since diminished centrifugal flow towards the uterus would increase pressure on the renal arteries, and the products of involution charge the circulation with matter which would cause irritation and contraction of arterioles, and so further increase arterial tension. The cold pale surface and dilated pupils pointed to superficial and cerebral anæmia from this cause, while the kidneys were in a state of active hyperæmia and the urine highly albuminous.

The treatment which I ordered was that the loins should be constantly poulticed, and that she should take directly a strong jalap purge, and twenty grains of bromide of potassium every three hours, the diet being restricted to milk. When I next saw her I was prepared to give a hypodermic injection of pilocarpine, which is a valuable remedy in such cases, causing profuse perspiration, with excessive excretion of urea. The girl, however, was so much better that she continued the former treatment with the very best results, and is now quite convalescent. Theoretically nitro-glycerine, or amyl nitrite, so valuable in exalted reflexes, should be of service in such a case. In my experience they cause dilatation of the contracted arterioles, and have decidedly a temporary, but no permanent, good effect. Seymour-street, $\mathrm{w}$.

\section{ON RETRACTION OF THE FLAP AFTER SYME'S AMPUTATION.}

\section{BY B EN E T T MAY, B.S.}

AlroNg the morbid conditions of stumps there is one incidentally alluded to by Mr. Savory in his "Notes on Surgery," which appeared in THE LANCET of Feb. 3rd. It is that of retraction of the heel flap after Syme's amputation. Although not generally recognised or looked for, it is, as he points out, a contingency to be provided against in certain cases, of which an example is now under my care at the Queen's Hospital. The patient is a young man on whom I performed a double amputation for a railway smash eighteen months ago, removing one limb below the knee and the other at the ankle. He made a very quick recovery, and for some time was very comfortable, but a few weeks ago he came to show me his Syme's stump, on which he was no longer able to bear any weight owing to the pain this gave him. I found that he was walking on the cicatix, where two large painful corns had developed, and that the heel flap, instead of receiving all the pressure, was retracted quite to the back of the leg, the integuments above it being thrown into several transverse folds from their laxity. It had evidently been drawn into this position by the muscles of the calf. Fortunately a division of tbe tendo Achillis has completely liberated the flap, and has enabled me to readjust it and replace it in normal position with complete relief to his troubles. I explain the occurrence in this case-Ist, by the fact, that the operation being a primary one, the muscles of the calf were caught in a state of full development and activity, and not attenuated by long disuse; and 2 nd, that owing to the rapid recovery which took place the tendo Achillis at a very early period attached itself to the heel flap-obviously a disadvantage, and one which I should endeavour to prevent in a future primary amputation by severing the tendon some distance above its insertion into the os calcis, or by removing an inch of it after division. The operation was originally performed almost precisely on the lines recommended by Mr. Savory, at least in the one particular of carrying the incision right across the sole and not backwards. Birmingham.

\section{ON A PROLONGED CASE OF TETANUS.}

UNDER THE CARE OF

DR. J. ROBINSON and MR. STENSON HOOKER.

Reported by the latter.

W. H- aged fourteen, on May 31st last, whilst bathing, received an incised wound on the ball of the great toe by treading on a piece of broken bottle; all went well until June 9th, when he complained of a "cold in the jaw," with stiffness of the muscles of the neck and between the shoulders; the wound was now healing nicely.-June 10th: The jaw is all but closed, and the muscles of the abdomen, back, and limbs contract still more strongly when touched. The urine is loaded with litbates; bowels rather confined. He takes liquid nourishment well, this being accomplished by suction, the teat of an infant's feeding bottle being squeezed between the teeth. Temperature $101 \cdot 6^{\circ}$; pulse regular, 100. Given twenty grains of bromide of potassium every four hours. 11th: There is general clonic spasm of all the voluntary muscles with intense pain, at times the condition is that known as opisthotonos. Given a scruple of chloral hydrate. Temperature $102 \cdot 2^{\circ}$; pulse $160 .-12$ th: Pain and muscular contraction still intense. Chloroform was administered, which relaxed the muscles and gave him sleep, but immediately on awakening the rigidity recurred. This extreme state continued. Chloroform being administered each day until the 23rd, there being no cessation from spasm except when under the influence either of this drug or of opiates. - 20th: Continues to take nourishment well; this consisted of milk soup, and beef-tea. Temperature $100 \cdot 2^{\circ}$ : pulse 100 . The bromide was evidently useless, and was therefore discontinued. - 23rd: Five drops of tincture of Calabar bean were given every two hours; the dose was increased gradually day by day. Wound healed. Pulse 116 ; temperature $100^{\circ} 2^{\circ}-26 \mathrm{th}$ : There is some loss of vision, which is hazy; he cannot distinguish objects further than a yard off. Pulse 124; temperature $1024^{\circ}$. Is now taking twenty drops of the drug every four hours. -27 th: There is complete loss of vision ; pupils are normal. $\mathrm{He}$ is slightly deliitous, and complains of tingling in both feet. There is no natural relaxation of the muscles. Pulse rather smaller, regular, 120 ; temperature $102^{\circ},-30$ th: The spasms are a little less violent and less frequent in the muscles of the trunk. He is now taking thirty drops for each dose. The vision has returned to some extent. Urine copious; bowels rather relaxed. Takes nourishment well. Pulse 94; temperature $100^{\circ}$.

July 3rd: The spasms are not so violent, and are confined to the lower extremities. He is now taking sixty-five drops every four hours. - 4th: The masseters relax slightly, and there is a constant desire to yawn. - 9th: Patient can now flex both arms, also the leg upon the thigh. Temperature $98 \cdot 8^{\circ}$; pulse 96 .

There was a slow improvement from this time, the intervals between the spasms becoming more and more prolonged. On the 12th he could eat solid food for the first time since the attack; and on the 3lst he could eat and talk fairly well; there was no spasm, but the legs were still somewhat rigid. On Aug. 5th all rigidity had disappeared, and the Calabar bean was omitted. The patient was up on the 7 th and complained only of general stiffness and debility, 